\title{
Vertebral Artery Syndrome
}

National Cancer Institute

\section{Source}

National Cancer Institute. Vertebral Artery Syndrome. NCI Thesaurus. Code C35123.

A syndrome which occurs as a result of the occlusion of one of the vertebral arteries. It may be caused by atherosclerosis, embolism or hemorrhage. Collateral circulation through the circle of Willis is usually comprised as well. Clinical signs may include vertigo, nystagmus, dysarthria, ataxia and sensorimotor deficits. Clinical course may lead to persistence of neurologic deficits. Prognosis is variable with a substantial risk for recurrent infarction. 